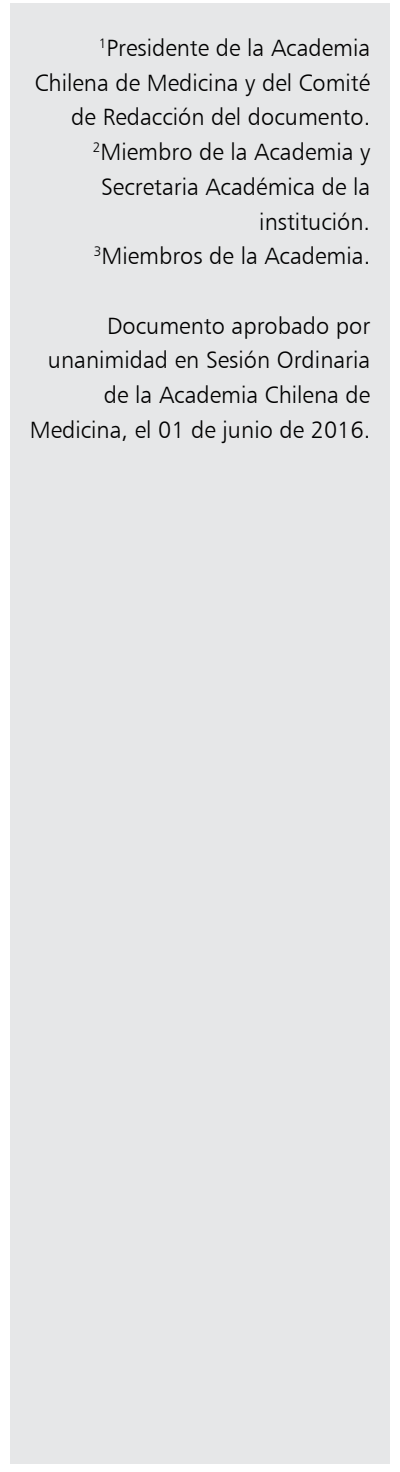

\section{Control de calidad de los médicos que inician el ejercicio profesional en Chile Declaración de la Academia Chilena de Medicina del Instituto de Chile}

\author{
COMITÉ DE REDACCIÓN: \\ HUMBERTO REYES B. ${ }^{1}$, COLOMBA NORERO V. ${ }^{2}$, \\ MARÍA EUGENIA PINTO C. ${ }^{3}$, OSVALDO LLANOS L ${ }^{3}$., \\ SERGIO MORÁN V. ${ }^{3}$, MIGUEL O'RYAN G. ${ }^{3}$, MARCELO WOLFF R. ${ }^{3}$
}

\section{Quality assurance of medical practice in Chile: Mandatory approval of the National Board Examination in Medicine as a quality assurance to start medical practice}

The Chilean Academy of Medicine supports the 2009 law by which all medical graduates from Chile as well as from foreign countries that wish to start their medical practice should have approved the National Board Examination in $\mathrm{Me}$ dicine ("Eunacom"). Success in this examination of basic clinical knowledge and competences is mandatory to become attending physicians at the Public Health Care System and to apply to state-funded postgraduate education. Annually, about $90 \%$ Chilean graduates approve this examination, while no more than $20 \%$ of foreign graduates do. Given the shortage of primary care physicians, some members of the Parliament have proposed to simplify the test or to exempt foreign medical graduates from it in order to broaden the coverage of primary care and decrease the waiting lists. The Chilean Academy of Medicine considers this shortcut strategy as dangerous for the Chilean population and unfair for our graduates. This Declaration has been send to the National Health Authorities, to Deans of all Medical Schools, and to public press media.

(Rev Med Chile 2016; 144: 656-657)

Key words: Education, medical; Licensure, medical; Professional practice; Quality assurance, health care.
$\mathrm{L}$ a opinión pública ha podido imponerse de iniciativas que, con la intención de corregir el déficit de médicos para la atención primaria de salud y de especialistas en el área pública, proponen la supresión o simplificación del Examen Único Nacional de Conocimientos de Medicina (EUNACOM) y la contratación masiva de médicos formados en el extranjero, sin evaluar sus conocimientos y competencias.

Considerando que el propósito fundamental de la Academia Chilena de Medicina es "contribuir al progreso del conocimiento médico en todos sus as- pectos, y aspira a ser la conciencia reflexiva de la medicina chilena", se emite la siguiente Declaración:

$1^{\circ}$ Es un imperativo ético para los médicos, sus instituciones y sus organizaciones velar por la calidad de la formación de quienes inician el ejercicio profesional.

$2^{\circ}$ Los países desarrollados, incluyendo los de la OCDE, exigen rendir exámenes nacionales o estatales para controlar la calidad de la formación de los médicos que se incorporan a la práctica profesional, con énfasis en el dominio 
del idioma local, en el conocimiento de las enfermedades más prevalentes en cada país y de las normativas sanitarias locales para su manejo. En varios países al examen nacional o estatal se agrega el requisito de una práctica controlada (internado y residencia) para poder ejercer la profesión o una especialidad determinada.

3o El aumento progresivo en el número de escuelas de medicina y de médicos egresados, hizo necesario que la Asociación de Facultades de Medicina Chilenas (ASOFAMECH), encargada por el Ministerio de Salud, implantara en el año 2009 un examen único nacional (EUNACOM) que evalúa conocimientos (sección teórica)y competencias mínimas necesarias para el ejercicio de la medicina (sección práctica).

$4^{\circ}$ La aprobación del EUNACOM es requisito legal para ser contratado en cargos médicos en el Sistema Nacional de Servicios de Salud, Consultorios Municipales y Fuerzas Armadas, para acceder a programas de especialización post título financiados por el Estado, y para firmar convenios de prestación de salud en modalidad de libre elección (FONASA). Este requisito aplica a los médicos titulados en universidades chilenas y a los médicos chilenos o extranjeros, titulados en universidades de otros países.

$5^{\circ}$ Los médicos titulados en universidades de otros países, han tenido un desempeño heterogéneo en este examen y su tasa de reprobación (variable anualmente entre 80 y $90 \%$ ) es notoriamente mayor que la de los médicos titulados en universidades chilenas (entre 6 y $10 \%$ ). Sus deficiencias en el examen práctico, que evalúa competencias clínicas, no puede justificarse aduciendo falta de familiaridad con la patología prevalente en nuestro país. Esto es motivo de preocupación para las entidades formadoras de médicos y atenta contra la fe pública ante el riesgo de una contratación indiscriminada de médicos para la atención primaria de salud, sin una supervisión permanente y eficaz de su desempeño. La Academia Chilena de Medicina considera inaceptable la contratación de médicos titulados en otros países, eximiéndolos de cumplir los requisitos exigidos a los médicos titulados en Chile.

$6^{\circ}$ El EUNACOM, como todo instrumento diseñado por el hombre, es susceptible de perfeccionamiento, siempre que no se altere su propósito fundacional. La Academia Chilena de Medicina considera indispensable mantener un control único nacional de la calidad de la formación de los médicos que inician el ejercicio profesional, porque "Están en juego la salud, el bienestar y la vida de las personas".

7o El desinterés de los médicos chilenos por desempeñarse en atención primaria resulta de la suma de factores no necesariamente ligados a su formación universitaria: la calidad de los recintos donde deberían trabajar, la disponibilidad de recursos tecnológicos propios del progreso de la medicina, el apoyo oportuno de laboratorios y de radiología, las expectativas salariales, las posibilidades de desarrollo profesional y de educación médica continuada. La corrección de estos factores es indispensable para conseguir el apego de los médicos a una actividad de alta relevancia nacional.

$8^{\circ}$ Los médicos chilenos han acogido siempre a los médicos extranjeros que, por diversos motivos, han migrado a nuestro país. En el Siglo XIX médicos europeos contribuyeron sustancialmente a elevar la calidad de la medicina nacional, la docencia universitaria y la salud pública. Desde el Siglo XX han ingresado al país muchos médicos formados en el extranjero, que han cumplido los requisitos legales exigidos en su momento y se han incorporado dignamente a la atención de salud, la docencia y la investigación, en las áreas pública y privada. La Academia Chilena de Medicina hace suya esta histórica y generosa disposición, siempre que se cumplan los requisitos mínimos de ingreso a la práctica profesional. 